\title{
COMPORTAMENTO ALIMENTAR E DIETA DE PHRYNOPS HILARII (DUMÉRIL \& BIBRON) EM CATIVEIRO (REPTILIA, TESTUDINES, CHELIDAE)
}

\author{
Flavio de Barros Molina 1, 2 \\ Mario Borges da Rocha ${ }^{1}$ \\ Luiz Antônio Beserra de Mello Lula ${ }^{1}$
}

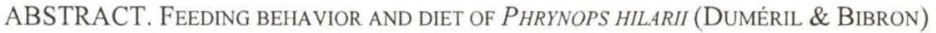
In CAPTIVITY (REPTILIA, TeSTudines, ChelidaE). Since 1985 the biology and behavior of Phrynops hilarii (Duméril \& Bibron, 1835) at São Paulo Zoo is being studied. Feeding behavior is divided in five phases (foraging, approach, capture, dilaceration and ingestion), but not necessarily all of them happen. During phase 1 the food or prey seems to be visually located. During phase 2 the food itens and stationary preys are approached and examined by olfaction. Moving preys are pursued and there is no olfactory examination. During phase 3 the food is captured by suction. When food is bigger than turtle mouth it is dilacerated by one or both forefeet used alternately (phase 4). Ingestion is accomplished by gradual suction (phase 5). Intra and inter-specific cleptoparasitism was observed. Success in capture and ingestion of food seems not be dependent on species or size of the turtle. Adults, young, and hatchlings of $P$. hilarii are primarily carnivorous and vegetables were rarely eaten.

KEY WORDS. Testudines, Chelidae, Phrynops hilarii, spotted-bellied side-necked turtle, feeding behavior, cleptoparasitism
\end{abstract}

Phrynops hilarii (Duméril \& Bibron, 1835) é espécie natural do rio Paraná e bacias adjacentes no sul do Brasil, Uruguai e norte da Argentina (IVERSON 1992), habitando riachos, lagos e brejos (ERNST \& BARBOUR 1989). Sua biologia é muito pouco conhecida e praticamente nada se sabe sobre a sua dieta e comportamento alimentar. Segundo FrEIBERG (1981), alimenta-se de peixes e moluscos, aceitando carne quando em cativeiro.

Com exceção das espécies que apresentam táticas de caça elaboradas, como a tartaruga aligator, Macroclemys temminckii (Harlan, 1835) (PRITCHARD 1984, 1989; CLOUDSLEY-THOMPSON 1994) e o matamatá, Chelus fimbriatus (Schneider, 1783) (Bellairs 1970; Pritchard 1984; Pritchard \& Trebbau 1984; CLOUDSLEY-THOMPSON 1994), poucas tiveram o seu comportamento alimentar descrito em detalhes.

Dentre as demais espécies da família Chelidae, vale ressaltar o trabalho de PRITCHARD (1984), que descreveu a tática de captura de peixes utilizada pelas

1) Setor de Répteis, Fundação Parque Zoológico de São Paulo. Avenida Miguel Stefano 4241, 04301-905 São Paulo, São Paulo, Brasil.

2) Bolsista do CNPq. 
espécies dos gêneros Hydromedusa Wagler, 1830 e Chelodina Fitzinger, 1826; o de Molina (1990), que analisou o comportamento alimentar de Phrynops geoffroanus (Schweigger, 1812) em cativeiro, comparando-o com o de outras espécies e gêneros neotropicais desta família; e o de SoUZA \& ABE (1995), que observaram a dieta e os hábitos alimentares de Hydromedusa maximiliani (Mikan, 1820) na natureza.

Em 1985, iniciou-se estudos no Zoológico de São Paulo, objetivando a reprodução de $P$. hilarii, bem como a aquisição de conhecimentos sobre a sua biologia e comportamento. Descreve-se aqui detalhadamente o seu comportamento alimentar e a dieta em cativeiro, dando continuidade ao trabalho de Molina (1990).

\section{MATERIAL \& MÉTODOS}

Os animais observados, 16 machos e 12 fêmeas adultas, 16 jovens e 5 recém-nascidos, pertencem ao acervo vivo do Setor de Répteis, Fundação Parque Zoológico de São Paulo, sendo provenientes da natureza, provavelmente do Rio Grande do Sul (Brasil).

Recintos a céu aberto, com ambiente terrestre e um tanque com água, foram utilizados na manutenção de adultos (recintos $n^{\circ} 78,37 \mathrm{~A}$ e 98 ) e jovens (recinto $\mathrm{n}^{\circ}$ 37A). Jovens e recém-nascidos também foram mantidos em tanques de fibro-cimento com área seca e alagada, mantidos a céu aberto durante os períodos da manhã e da tarde. A temperatura da água variou entre 15 e $30^{\circ} \mathrm{C}$ nos tanques dos recintos e entre 18 e $30^{\circ} \mathrm{C}$ nos tanques de fibro-cimento. Os recintos foram descritos por MOLINA \& SAJDAK (1993). Adultos, jovens e recém-nascidos conviveram com outras espécies de quelônios e de jacarés.

A dieta oferecida nos recintos e tanques com $P$. hilarii era elaborada para atender as necessidades de todas as espécies que aí habitavam. Consistia de peixes, carne bovina (músculo), tomate, cenoura, frutas (banana, mamão e laranja) e verduras (couve, almeirão e repolho). Os alimentos eram cortados em tamanhos adequados, complementados com farinha de osso e servidos cru. Os adultos tiveram acesso aos pintos oferecidos para os jacarés e os filhotes receberam periodicamente minhocas, Pheretima sp., larvas e adultos do besouro da farinha, Tenebrio molitor Linnaeus, 1758 e Alphitobius diaperinus (Panzer, 1797) e ninfas e adultos de grilo, Gryllus sp., estes complementados com fosfato de tricálcio. Apenas os invertebrados foram oferecidos vivos. A alimentação era servida na água, cinco vezes por semana, sempre as 9:30h. Durante as épocas frias do ano, geralmente no final do outono e durante o inverno, a alimentação nos recintos era oferecida apenas três vezes por semana.

A descrição do comportamento alimentar baseou-se em um total aproximado de 150 horas de observações intermitentes, realizadas principalmente durante 0 horário da alimentação e com maior frequência nos anos de 1987, 1988 e 1993. Foi utilizado o método da amostragem de todas as ocorrências de um dado comportamento (sensu ALTMANN 1974). 


\section{RESULTADOS}

O comportamento alimentar, observado sempre dentro da água, divide-se em cinco etapas sucessivas, nem todas obrigatórias: forrageio, aproximação, apreensão, dilaceração e ingestão do alimento.

Durante a primeira etapa, o indivíduo desloca-se pelo tanque, com movimentos lentos, pescoço e cabeça esticados. $\mathrm{O}$ fato destes cágados localizarem pedaços de carne e frutas situados a mais de um metro de distância sugere fortemente que a localização do alimento seja visual.

Após a localização, inicia-se a segunda etapa, que se caracteriza por uma aproximação com movimentos lentos, se $o$ alimento estiver imóvel (e.g. pedaços de carne e frutas) ou por uma perseguição, se o alimento for uma presa viva dotada de agilidade (e.g. grilos). O pescoço continua esticado e a cabeça direcionada ao alimento. A aproximação pode ser feita a alimentos em qualquer nível da coluna d'água.

No final da segunda etapa, o cágado aproxima as narinas do alimento, realizando um reconhecimento olfativo. Se o alimento o interessar (e.g. carne e peixe), será apreendido, caso contrário (e.g. frutas), voltará à primeira etapa. O reconhecimento olfativo não ocorre quando a segunda etapa caracteriza-se como uma perseguição.

A terceira etapa tem início quando o alimento encontra-se dentro do raio de ação do cágado. Normalmente, com o pescoço encurvado, o animal projeta a cabeça com um rápido movimento em direção ao alimento, abrindo a boca quando próximo a este, a fim de succioná-lo. A apreensão do alimento também pode ocorrer em qualquer nível da coluna d'água.

A quarta etapa ocorre apenas quando o alimento é maior que a cabeça do cágado. A dilaceração é feita com auxílio das mãos, nunca utilizadas simultaneamente. A mão utilizada é a que fica do lado para o qual a cabeça é recolhida e ao mesmo tempo em que esta é retraída para junto do casco, a mão é afastada em arco, com as garras puxando parte do alimento. Os movimentos podem ser repetidos várias vezes, antes de ocorrer alternação da mão. Quando o alimento é menor que a cabeça do cágado, ele é ingerido imediatamente após a apreensão.

A ingestão ocorre por sucção gradual, uma vez que ao fechar a boca o alimento tende a ser expelido. O cágado abre a boca várias vezes, succionando cada vez mais o pedaço abocanhado, até ingerí-lo por completo. Cada vez que fecha a boca, ocorre expulsão da água succionada e restos de alimento (e.g. élitros de coleópteros).

Nas inúmeras ocasiões em que o comportamento alimentar foi observado, ocorreu disputa pelo alimento entre indivíduos de $P$. hilarii, ou entre estes e exemplares de $P$. geoffroanus e Trachemys dorbignyi (Duméril \& Bibron, 1835). Nestas disputas, que envolviam exemplares de diferentes tamanhos, os cágados demonstravam grande interesse em capturar o alimento já apreendido por outro indivíduo, retirando-o tanto das suas mãos, como da sua boca (Cleptoparasitismo sensu HAYES 1987). Por isso, quando muitos indivíduos alimentavam-se próximos, aquele que obtinha um pedaço grande de alimento procurava afastar-se, para não 
perdê-lo parcial ou totalmente. As etapas subsequentes eram finalizadas em local mais sossegado ou durante o afastamento, quase sempre perseguido pelos outros quelônios. O sucesso na apreensão e ingestão do alimento não pareceu depender do tamanho ou espécie do indivíduo.

Adultos e jovens de $P$. hilarii mostraram-se primariamente onicarnivoros, raramente ingerindo pequenos pedaços dos vegetais oferecidos. Aceitaram todos os alimentos de origem animal, sendo que os adultos tentaram abocanhar inclusive os pintos. As minhocas e larvas do besouro da farinha foram bastante apreciadas. Os grilos foram agilmente perseguidos e geralmente capturados. Não foi observada variação ontogenética no comportamento alimentar ou na dieta ingerida pela espécie.

\section{DISCUSSÃO}

As observações aqui realizadas mostram que $P$. hilarii apresenta o mesmo padrão de comportamento alimentar descrito por Molina (1990) para P. geoffroanus, estando as duas espécies taxonomicamente muito próximas (RHODIN \& MITTERMEIER 1983). Isto demonstra que, apesar da ocorrência de especializações, como as descritas por BELKIN \& GANS (1968), BELLAIRS (1970) e PRITCHARD (1984), o comportamento alimentar dos quelônios mostra-se de forma geral bastante estereotipado, conforme já havia sido comentado por MoLINA (1990).

Segundo Molina (1990), Acanthochelys spixii (Duméril \& Bibron, 1835), Acanthochelys radiolata (Mikan, 1820), Platemys platycephala (Schneider, 1792), Hydromedusa tectifera Cope, 1869 e H. maximiliani (espécies da família Chelidae) exibem comportamento alimentar similar ao de $P$. geoffroanus, embora por não serem tão ágeis, encontrem maiores dificuldades para caçar animais de movimentos rápidos, como grilos.

O comportamento alimentar de $H$. maximiliani na natureza foi observado por Souza (comunicação pessoal). Durante o forrageio, o cágado esquadrinha minuciosamente o substrato, descrevendo um ângulo de $180^{\circ}$ com movimentos laterais do pescoço. Após ser localizada, a presa era capturada com um rápido movimento do pescoço, comportamento bastante semelhante ao descrito por MoLINA (1990) para $P$. geoffroanus.

A apreensão do alimento descrita para $P$. hilarii e para $P$. geoffroanus (Molina 1990) mostrou-se muito semelhante à tática de captura de peixes descrita por PRITCHARD (1984) e denominada de estocada (spear-fishing). Segundo o autor, entre os membros da família Chelidae, esta tática é utilizada pelas espécies dos gêneros Chelodina e Hydromedusa.

FRETEY (apud PRITCHARD \& TREBBAU 1984) observou Phrynops nasutus (Schweigger, 1812) obtendo alimento por sucção e PRITCHARD \& TREBBAU (1984) comentaram que o extremo desenvolvimento do hióide nesta espécie sugere adaptações paralelas às exibidas por $C$. fimbriatus.

Entre as espécies de outras famílias que utilizam a tática de estocada estão Chelydra serpentina (Linnaeus, 1758) (Chelydridae; Froese \& BURGHARDT 1974; PRITCHARD 1984), Deirochelys reticularia (Latreille, 1801) (Emydidae; PRITCHARD 1984) e Chitra indica (Gray, 1831) (Trionychidae; PRITCHARD 1984). 
As mesmas etapas do comportamento alimentar de $P$. hilarii e $P$. geoffroanus (Molina 1990) foram observadas em espécies de outras famílias (Pelomedusidae, Emydidae e Kinosternidae), sendo que nestas, a apreensão do alimento ocorre principalmente por um abocanhamento, e não pela rápida projeção da cabeça seguida de sucção (MolinA 1990). A sucção ocorre em menor grau, auxiliando apenas na ingestão de pequenas partículas (MONGE-NÁJERA \& MOREVA-BRENES 1987; Molina 1990). Durante a dilaceração, espécies da subordem Cryptodira utilizam as mãos alternada ou simultaneamente (MOLINA 1990).

Ao contrário do descrito por BELMONTE (1991), não foi observada a realização de limpeza bucal em $P$. hilarii, mesmo quando os animais se alimentaram de minhocas. Molina (1990) também não observou esta etapa no comportamento alimentar de $P$. geoffroanus e dos demais quelônios que estudou.

A visão e o olfato encontram-se bem desenvolvidos nos quelônios (CAMPBEll 1967; Bellairs \& AtTRIDGE 1978; MANTON 1979; SPELlERBERG 1982; WELDON et al. 1994), parecendo ser os únicos sentidos envolvidos no processo de localização e reconhecimento do alimento em $P$. hilarii. É provável que em águas turvas o alimento seja localizado principalmente através do olfato, como ocorre com H. maximiliani (Souza comunicação pessoal). BELMONTE (1991) acredita que as barbelas da região gular de $P$. hilarii também possam ser de importância para a obtenção do alimento. Visão e olfato são importantes na localização e reconhecimento de alimento em várias outras espécies (BELKIN \& GANS 1968; MAHMOUD 1968; Hulse 1974; Pritchard 1984; Pritchard \& Trebbau 1984; Molina 1990).

Foi observada a ocorrência de cleptoparasitismo intra e inter-específico (com $P$. geoffroanus e $T$. dorbignyi) durante a alimentação, envolvendo indivíduos de diferentes tamanhos. Segundo Hayes (1987), apesar de tal comportamento ser pouco relatado na bibliografia, parece ocorrer com frequência em várias espécies de répteis mantidos em cativeiro. Cleptoparasitismo intra e inter-específico foi observado em $P$. geoffroanus, sendo que o sucesso na apreensão e ingestão do alimento também não pareceu depender do tamanho ou espécie do indivíduo (MOLINA 1990).

Adultos e jovens de $P$. hilarii mostraram-se primariamente onicarnívoros, como os demais membros da família, considerados primariamente (MEDEM 1966; MitTERMEIER et al. 1978; Molina 1990), ou totalmente carnívoros (MEDEM 1960; Pritchard 1984; Pritchard \& TREBbAu 1984; SouzA \& ABE 1995).

Phrynops hilarii não apresentou variação ontogenética no comportamento alimentar e nem na dieta, ao contrário do que ocorre respectivamente em $C$. serpentina (FROESE \& BURGHARDT 1974) e em Trachemys scripta (Schoepff, 1792) (Clark \& GibBONS 1969; HART 1983).

AGRADECIMENTOS. Ao diretor da Fundação Parque Zoológico de São Paulo, Dr. Adayr Mafuz Saliba, e aos demais colegas desta Fundação, pelo apoio e incentivos constantes. Aos Drs Ana Maria de Souza Oliveira, Miguel Trefaut Rodrigues, Norma Gomes e Sérgio de Almeida Rodrigues, pelas discussões referentes ao comportamento alimentar e dieta dos 
quelônios. Ao Roberto da Rocha e Silva, pelas sugestões feitas ao manuscrito e ao Richard A. Sajdak, pelo envio de importante material bibliográfico. Ao Franco Leandro de Souza, pelas comunicações pessoais. Ao Conselho Nacional de Desenvolvimento Científico e Tecnológico (CNPq), pela Bolsa de Pesquisa concedida à F.B. Molina (processo 300328/95-8).

\section{REFERÊNCIAS BIBLIOGRÁFICAS}

ALtMANN, J. 1974. Observational study of behavior: Sampling methods. Behaviour 49 (3-4): 227-267.

BELKIN, D.A. \& C. GANS. 1968. An unusual chelonian feeding niche. Ecology 49 (4): 768-769.

Bellairs, A. 1970. The life of reptiles. New York, Universe, vol. 1, XII+282p. Bellairs, A. \& J. AtTridge. 1978. Los reptiles. Madrid, H. Blume, 261p.

BELMONTE, F.T. 1991. Observaciones sobre el comportamiento de Phrynops hilarii (Dumeril \& Bibron) (Testudines - Chelidae) en los ocho primeros meses de vida. Acta zool. lilloana 40 (1): 5-12.

CAMPBELL, H.W. 1967. The turtle in modern research. "How well will the turtle adapt to the moon's conditions?" Int. Turtle Tortoise Soc. 1: 10-12.

Clark, D.B. \& J.W. GibBons. 1969. Dietary shift in the turtle Pseudemys scripta (Schoepff) from youth to maturity. Copeia 1969 (4): 704-706.

Cloudsley-Thompson, J.L. 1994. Predation and defence amongst reptiles. Taunton, R \& A Publishing Limited, VIII+138p.

ERnSt, C.H. \& R.W. BARbour. 1989. Turtles of the world. Washington, Smithsonian Institution Press, XII+313p.

FreiberG, M.A. 1981. Turtles of South America. Neptune City, T.F.H. Publications, $125 \mathrm{p}$.

Froese, A.D. \& G.M. Burghardt. 1974. Food competition in captive juvenile snapping turtles, Chelydra serpentina. Anim. Behav. 22 (3): 735-740.

HART, D.R. 1983. Dietary and habitat shift with size of red-eared turtles (Pseudemys scripta) in a southern Louisiana population. Herpetologica 39 (3): 285-290.

HAYES, F.E. 1987. Intraspecific kleptoparasitism and aggression in young, captive red-eared sliders (Pseudemys scripta elegans). Bull. Md Herpetol. Soc. 23 (3): 109-112.

HulsE, A.C. 1974. Food habits and feeding behavior in Kinosternon sonoriense (Chelonia: Kinosternidae). Jour. Herpet. 8 (3): 195-200.

IVERSON, J.B. 1992. A revised checklist with distribution maps of the turtles of the world. Indiana, Ed. by the author, XIII+363p.

MAHMOUD, I.Y. 1968. Feeding behavior in kinosternid turtles. Herpetologica 24 (4): 300-305.

MANTON, M.L. 1979. Olfaction and behavior, p. 289-301. In: M. HARLESS \& H. MORLOCK (Eds). Turtles: Perspectives and research. New York, John Wiley and Sons, XIV+695p.

MEDEM, F. 1960. Informe sobre reptiles colombianos. (V) Observaciones sobre la distribucion geografica y ecologia de la tortuga Phrynops geoffroana ssp. en 
Colombia. Noved. Colombianas 1 (5): 291-300.

MEDEM, F. 1966. Contribuciones al conocimiento sobre la ecologia y distribución geográfica de Phrynops (Batrachemys) dahli; (Testudinata, Pleurodira, Chelidae). Caldasia 9 (45): 467-489.

Mittermeier, R.A.; A.G.J. Rhodin; F. Medem; P. Soini; M.S. Hoogmoed \& N. CARRILlo de ESPINOZA. 1978. Distribution of the South American chelid turtle Phrynops gibbus, with observations on habitat and reproduction. Herpetologica 34 (1): 94-100.

MolinA, F.B. 1990. Observações sobre os hábitos e o comportamento alimentar de Phrynops geoffroanus (Schweigger, 1812) em cativeiro (Reptilia, Testudines, Chelidae). Revta bras. Zool. 7 (3): 319-326.

MolinA, F.B. \& R.A. SAJDAK. 1993. Observações sobre a preferência térmica e o comportamento de termorregulação no jacaré-de-papo-amarelo, Caiman latirostris, em cativeiro: Variações ontogenéticas e algumas comparações com outras espécies de jacarés neotropicais, p. 93-132. In: L.M. VERDADE; I.U. Packer; M.B. Rocha; F.B. Molina; P.G. Duarte \& L.A.B.M. Lula (Eds). Anais do $3^{\circ}$ Workshop sobre Conservação e Manejo do Jacaré-de-papoamarelo (Caiman latirostris). Piracicaba, ESALQ/USP, 220p.

Monge-NÁJERA, J. \& B. Moreva-BrenEs. 1987. Notes on the feeding behavior of a juvenile mud turtle Kinosternon scorpioides. Herpetol. Rev. 18 (1): 7-8.

PRITCHARD, P.C.H. 1984. Piscivory in turtles, and evolution of the long-necked Chelidae. Symp. zool. Soc. Lond. (52): 87-110.

1989. The alligator snapping turtle: Biology and conservation. Milwaukee, Milwaukee Public Museum, XI+104p.

Pritchard, P.C.H. \& P. TrebBau. 1984. The turtles of Venezuela. Oxford, Society for the Study of Amphibians and Reptiles, VIII+403p.

RHODIN, A.G.J. \& R.A. MitTERMEIER. 1983. Description of Phrynops williamsi, a new species of chelid turtle of the South American $P$. geoffroanus complex, $\mathrm{p}$. 58-73. In: A.G.J. RHODIN \& K. MiYATA (Eds). Advances in herpetology and evolutionary biology. Essays in honor of Ernest E. Williams. Cambridge, Mass., Museum of Comparative Zoology, 725p.

SouZA, F.L. \& A.S. ABE. 1995. Observations on feeding habits of Hydromedusa maximiliani (Testudines: Chelidae) in Southeastern Brazil. Chelonian Conservation and Biology 1 (4): 320-322.

SPELLERBERG, I.F. 1982. Biology of reptiles: An ecological approach. Glasgow, Blackie \& Son Ltd, VIII+158p.

Weldon, P.J.; B.J. Demeter; T. Walsh \& J.S.E. Kleister. 1994. Chemoreception in the feeding behavior of reptiles: considerations for maintenance and management, p.61-70. In: J.B. MurPhy; K. AdLER \& J.T. Collins (Eds). Captive management and conservation of amphibians and reptiles. Ithaca, Society for the Study of Amphibians and Reptiles, 408p.

Recebido em 10.I.1997; aceito em 05.XI.1997. 